\title{
Durvalumab in frail and elder patients with stage four NSCLC: Study protocol of the randomized phase II DURATION trial
}

\section{Jonas Kuon ( $\boldsymbol{\nabla}$ jonas.kuon@med.uni-heidelberg.de )}

Thoraxklinik-Heidelberg gGmbH https://orcid.org/0000-0002-8566-1099

\section{Adriane Hommertgen}

German Cancer Research Center, Abteilung für Radioonkologie

\section{Johannes Krisam}

Heidelberg University Hospital, Institute of Medical Biometry and Informatics

\section{Felix Lasitschka}

Heidelberg University Hospital, Institute of Pathology, Translational Lung Research Center Heidelgerg TLRC-H, Member of the German Center for Lung Research DZL

\section{Albrecht Stenzinger}

Heidelberg University Hospital, Institute of Pathology, Translational Lung Research Center Heidelberg TLRC-H, Member of the German Center for Lung Research DZL

\section{Miriam Blasi}

Thoraxklinik-Heidelberg gGmbH

\section{Farastuk Bozorgmehr}

Heidelberg University Hospital, Department of Thoracic Oncology, Translational Lung Research Center Heidelberg TLRC-H, Member of the German Center for Lung Research DZL

\section{Martin Maenz}

AlO-Studien-gGmbH Berlin

\section{Meinhard Kieser}

Heidelberg University Hospital, Institute of Medical Biometry and Informatics

\section{Marc Schneider}

Heidelberg University Hospital, Translational Research Unit, Translational Lung Research Center Heidelberg TLRC-H, Member of the German Center for Lung Research DZL

\section{Michael Thomas}

Heidelberg University Hospital, Department of Thoracic Oncology, Translational Lung Research Center Heidelberg TLRC-H, Member of the German Center for Lung Research DZL

\section{Study protocol}

Keywords: Lung Cancer; Durvalumab; PD-L1; elderly; frail; CARG; 
Posted Date: March 12th, 2020

DOI: https://doi.org/10.21203/rs.2.16770/v2

License: (c) (i) This work is licensed under a Creative Commons Attribution 4.0 International License. Read Full License 


\section{Abstract}

Background: Elderly patients represent a major fraction of non-small cell lung cancer (NSCLC) patients in routine clinical practice, but they are still underrepresented in clinical trials. In particular, data regarding efficacy and safety in frail or elderly patients with respect to immunotherapy are lacking. Importantly, immunosenescence in elderly patients might interfere with activities of immune-modulating drugs such as PD-1/PD-L1 inhibitors. Thus, there is an urgent need to assess safety and efficacy of such inhibitors in this group. Methods/design: In this prospective, open label, treatment stratified, and randomized phase II study, 200 patients with stage IV NSCLC amenable at least to single-agent chemotherapy (CT). Eligible patients must be 70 years or older and/or "frail" (Charlson Comorbidity Index $>1$ ) or have a restricted performance status (Eastern Cooperative Oncology Group, ECOG >1). Patients are stratified according to modified Cancer and Age Research Group (CARG) score:"fit" patients are allocated to combination CT (carboplatin/ nab -paclitaxel), "less fit" patients receive single-agent CT (gemcitabine or vinorelbine). After allocation to strata, patients are randomized 1:1 to receive either 4 cycles of CT or 2 cycles of CT followed by 2 cycles of durvalumab and subsequent maintenance treatment with durvalumab every 4 weeks. The primary endpoint is the rate of treatment related grade III/IV adverse events (Common Terminology Criteria for Adverse Events, CTCAE V4.03). As secondary endpoints, progression-free survival (PFS) according to Response Evaluation Criteria in Solid Tumours (RECIST) version 1.1, response rate (RR), overall survival (OS), descriptive subgroup analyses according to PD-L1 expression, and quality of life are addressed. Geriatric screening assessments and functional tests will be performed to complete the phenotyping of a potential "frail" and "elderly" patient cohort. The trial is accompanied by a biomaterial repository to explore potential biomarkers. Discussion: The DURATION trial will prospectively investigate the safety and tolerability of anti-PD-L1 treatment with durvalumab after chemotherapy in elderly and frail patients and thereby provide new insights into the effect of PD-L1 blockade and the impact of immunosenescence in this cohort of patients.

\section{Background}

Lung cancer is one of the most commonly diagnosed cancer types and the leading cause of cancer death worldwide (1). Lung cancer is predominantly a disease of the elderly with about $50 \%$ of patients diagnosed aged 70 years or older (2). Age is an independent prognostic factor affecting survival in this group of patients (3).

Metastatic non-small cell lung cancer (NSCLC) carries a dismal prognosis with a median survival of less than 12 months (4). Systemic treatment, such as mono-chemotherapy (e.g. vinorelbine or gemcitabine) $(5,6)$ or a dose adapted combination of carboplatin (AUC6) / paclitaxel $(90 \mathrm{mg} / \mathrm{m} 2 ; \mathrm{d} 1+8+15)(7)$ is considered to improve outcome in patients who are aged $\geq 70$ years, are frail or have a reduced performance score (i.e., ECOG $\geq 2$ ).

The use of geriatric assessment and screening tools is recommended in elderly patients to better adapt intensity of treatment to patient condition and comorbidities (8). As a complex geriatric assessment (comprehensive geriatric assessment) is time- and resource-consuming and potentially not required for all 
patients, shorter pre-therapy risk assessments have been developed, i.e. the CRASH (Chemotherapy Risk Assessment Scale for High-Age Patients) (9) and the CARG (Cancer and Age Research Group) score (10).

While the CRASH score contains variables for clinical and geriatric assessment, the CARG score comprises geriatric assessment questions and clinical questions concerning items retrieved from everyday practice. Both scores are considered as useful toxicity prediction tools, appropriate for implementation in routine clinical practice, with a potential impact on the optimization of therapy selection for elderly patients with cancer (11).

With the advent of immune-oncology, new options have become available. Currently, checkpoint inhibition therapies targeting PD-1 and PD-L1 are established as 1 $^{\text {st }}$-line treatment in metastatic lung cancer. This is based on several impressive efficacy results of anti-PD-1/PD-L1 antibodies in clinical trials in NSCLC that have led to the approval of nivolumab, pembrolizumab and atezolizumab (12-15) first in advanced therapy lines and later in the $1^{\text {st }}$ line setting (mono-immunotherapy for PD-L1 $\geq 50 \%$ or regardless of PD-L 1 tumor expression in combination with a combination chemotherapy) $(16,17)$. However, clinical evidence about the tolerability and safety of checkpoint inhibition as a treatment option in frail and elderly patients is still lacking.

In addition, it remains an unresolved question whether efficacy of checkpoint inhibitors in older patients is affected by a still poorly characterized phenomenon referred to as immunosenescence, i.e. the global and progressive remodeling of immune functions with aging $(14,15,18,19)$. Age-related alterations such as, for example, impaired $\mathrm{T}$ cell activation, reduced $\mathrm{T}$ cell receptor diversity, altered antigen-uptake and -

presenting functions, or the increased generation of immune suppressive cells, may impair the antitumor response and thus could be one of the reasons for a higher incidence and prevalence of most cancers in older people.

Furthermore, a paradoxical stimulation of tumor growth upon initiation of treatment with checkpoint inhibitors, the so-called hyperprogressive disease, has been reported in a recent retrospective analysis in up to $19 \%$ of patients older than 65 years. This could potentially be caused by an altered immune function due to immunosenescence (20).

In conclusion, with the increasing use of immunotherapy in everyday clinical practice there is a growing interest in immunosenescence and how it may correlate with outcomes of immunotherapy in elderly patients $(18,21)$. However, data derived from randomized trials are lacking to investigate safety and tolerability of checkpoint inhibitors in this specific population of lung cancer patients.

\section{Methods/design}

\section{Study design}

The DURATION study is an open label, treatment stratified, and randomized phase II study (Figure 1) enrolling patients with histologically confirmed NSCLC (adenocarcinoma and squamous) stage four 
(metastatic) prior to any systemic treatment. All procedures and time frames displaced in figure 1 and schedule of assessment (Table 3 ) are developed according to the Standard Protocol Items:

Recommendations for Interventional Trials (SPIRIT). Additional file 1 contains the complete SPIRIT checklist.

\section{Study setting}

The DURATION trial is a multicenter trial, recruiting patients from approximately 30 sites across Germany. A full list of sites can be obtained at clinicaltrials.gov (NCT03345810).

\section{Study objectives}

\section{Primary objective}

The primary objective is to investigate the safety and tolerability of sequential therapy consisting of standard of care single agent or doublet chemotherapy followed by durvalumab in comparison to standard of care single-agent or doublet chemotherapy in frail and/or elderly patients.

\section{Secondary objectives}

Secondary objectives are to collect information on efficacy, safety and quality of life parameters and to investigate the utility of geriatric assessments for treatment guidance.

\section{Exploratory objectives}

Exploratory objectives are to identify potential predictive biomarkers for efficacy variables. To this end, tissue collection and blood sampling will be performed before and during course of disease/treatment. The blood and tissue samples will be subjected to molecular analyses to search for markers of immune response in this population.

Analysis of biomarker data will include correlations with clinical phenotype and tumor PD-L1 expression.

\section{Characteristics of patients}

200 frail and/or elderly patients with metastatic non-small cell lung cancer with no targetable molecular alterations (EGFR $\left.{ }^{\mathrm{wt}}, E M L 4 A L K^{\text {transl-}}\right)$ before $1^{\text {st }}$-line treatment will be included.

Key inclusion criteria are age $\geq 70$ years and/or $\mathrm{CCl}>1$ and/or ECOG $>1$, previously untreated NSCLC with no targetable molecular alterations ( $\left.E G F R^{\mathrm{wt}}, E M L 4 A L K^{\text {transl-}}\right)$ and the availability of a formalin-fixed, paraffin-embedded (FFPE) tumor tissue block (fresh or archival less than three years old or recent) or a minimum of ten unstained slides of tumor sample for biomarker (PD-L1) evaluation. Key exclusion criteria include mixed small cell lung cancer and NSCLC histology, and history of another primary active malignancy or active autoimmune disease.

For a full list of inclusion and exclusion criteria see Table 1. 


\section{Procedures for stratification}

Patients are stratified by the principal investigator or authorized delegate from the study staff according to modified Cancer and Age Research Group (CARG) to receive respectively $(10,22)$ :

- Total risk score $\leq 3 \rightarrow$ doublet chemotherapy

- Total risk score $>3 \rightarrow$ single-agent chemotherapy

The aim is to prevent $>50 \%$ of standard chemotherapy toxicities (CTCAE grade III/IV). The risk score will be determined according to Table 2.

\section{Study procedures}

The subjects must first read, understand, and sign the -approved informed consent form (ICF ) before any study-specific screening procedures are performed. After signing the ICF, completing all screening procedures, and being deemed eligible for entry, subjects will be enrolled in the study. Procedures that are performed prior to the signing of the ICF and are considered standard of care may be used as screening assessments if they fall within the 28-day screening window (in particular tumor stagings).

After the stratification procedure, (modified CARG score) done by the investigator, the investigator will access the randomisation website to assign the participant to the treatment arms. Randomisation is performed using permuted block randomisation with fixed block lengths. Treatment arm allocation (ratio 1:1) will be done following the Standard Operational Procedures of the Institut für Klinische Forschung (IKF, Frankfurt Germany), which is the clinical research organisation of the DURATION trial (CRO). After randomisation the system will immediately confirm the patients allocation to the treatment arms, to receive either four cycles of single agent or doublet chemotherapy or two cycles of single-agent or doublet chemotherapy followed by two cycles of immunotherapy. After four cycles of standard chemotherapy patients receive either follow-up care (arm $A$ and $D$ ) or a maintenance therapy with durvalumab for a maximum of two years in the experimental arms $B$ and $C$. Dose modification and toxicity management is described in detail in the chapter "Treatment plan" of the protocol. Furthermore, detailed information about permitted or prohibited concomitant treatment are obtained in the protocol.

Subject adherence to protocol interventions: No particular methods to improve adherence to trial intervention have been implemented. Due to the nature of the disease under study (advance or metastatic lung cancer), patients are naturally motivated to adhere to the trial intervention. Furthermore, the trial medication is not self-administered by the study subjects but rather investigator administered according to a pre-specified visiting calendar of the trial protocol.

Strategies to achieve target sample size: The DURATION trial was set-up with the clinical trial network of the Arbeitsgemeinschaft Internistische Onkologie (AIO). Each of the 30 participating site was selected by the sponsor based on former and anticipated accrual performance. The accrual rate is monitored on a monthly basis and any shortfall communicated to the $\mathrm{Cl}$ and the sponsor. Regular newsletters and 
meetings within the trial network are routine tools to maintain a steady accrual rate. Based on the discretion of the sponsor additional study sites may be included to bolster recruitment.

For each patient enrolled, an electronic case report form (eCRF) must be completed by the principal investigator or authorized delegate from the study staff. This also applies to records for those patients who fail to complete the study. If a patient withdraws from the study, the reason must be noted in the eCRF. Subjects who are permanently discontinued from the study medication will be followed for safety unless consent is withdrawn or the subject is lost to follow-up or enrolled in another clinical study. All subjects will be followed for survival. Subjects who decline to return to the site for evaluations will be offered follow-up by phone as an alternative.

Treatment emergent adverse events (AEs) according to common terminology criteria for adverse events (CTCAE) version 4.03 will be recorded in the eCRF using a recognized medical term or diagnosis that accurately reflects the event. Adverse events will be assessed by the investigator for severity, relationship to the investigational product, possible etiologies, and whether the event meets criteria of a serious adverse event (SAE) and therefore requires immediate notification to the CRO. AEs and SAEs will be recorded during the entire study duration, including the regular 30 day safety follow-up period after the end-of-treatment (EOT) visit. Subsequently, subjects will be followed for ongoing study treatment-related adverse events until resolved, return to baseline or deemed irreversible, until lost to follow-up, or withdrawal of study consent. Non-serious adverse events are recorded from time of signed informed consent until 30 days after last dose of the investigational medicinal product (IMP). Serious adverse events are recorded from time of signed informed consent until 90 days after last dose of IMP. Adverse events of special interest (non-serious and serious AESI) are recorded from time of signed informed consent until 90 days after last dose of IMP. The investigator is responsible for ensuring that all adverse events observed by the investigator or reported by patient are properly captured in the patients' medical records. During the course of the study all AEs and SAEs should be proactively followed up for each subject. Every effort should be made to obtain a resolution for all events, even if the events continue after discontinuation/study completion.

A data safety monitoring board (DSMB) is installed to monitor trial conduct. The primary objective of the DSMB is to monitor the safety of the intervention of the clinical study according to the protocol. The DSMB will evaluate the safety of the study intervention and will propose changes, termination or continuation of the trial to the sponsor and the Coordinating Investigator. It will consist of two experienced thoracic oncologists. The first interim safety assessment will be conducted after the first 20 subjects have been treated with at least two cycles of durvalumab. Thereafter, yearly assessments, synchronized with the annual safety reports, will be performed. Details are provided in the protocol referring to the DSMB Charter.

Monitoring/audits: The CRO must provide a trained monitor to assist the investigators in conducting the clinical study. The monitor has the responsibility of reviewing the ongoing study with the investigators to verify adherence to the protocol and to deal with any problems that arise. The study monitor will review the eCRF data for completeness and accuracy during the monitoring visits. The study monitor will point out 
any discrepancies between source data and the data captured in the eCRF. The monitor will issue electronic queries to site staff to initiate discrepancy resolution. Discrepancies which require eCRF data corrections have to be re-solved by authorized site personnel by answering these monitoring queries. The frequency of on-site visits will depend on the number of recruited patients and results of prior monitorings (risk-adapted monitoring). The monitor must be given access to subject medical records and other studyrelated records needed to verify the entries in the eCRF. The Investigator agrees to cooperate with the monitor to ensure that any problems detected in the course of these monitoring visits, including delays in completing case report forms, are resolved. The Investigator has to ensure that all data required according to this protocol will be entered promptly in the eCRF.

Collection of Safety data / harms: It is the responsibility of the investigators to document all adverse events occurring during the study in the patients' medical files and the eCRF. Any serious adverse event (SAE), AE of special interest, overdose of IMP and pregnancies must be reported immediately (within 24 hours) to the sponsor. The Sponsor and the $\mathrm{CRO}$ will ensure compliance with all regulatory reporting requirements including the notification of the appropriate Ethics Committees, Competent Authority and participating investigators of all serious adverse events occurring at the sites in accordance with national law, ICH Good Clinical Practice and European / EMA requirements.

A Sponsor representative (e.g. CRO) will medically review all SAE reports and perform the expectedness assessment.

Every SAE, being assessed by either the investigator or the Sponsor as suspected to be related to IMP und assessed as being either unexpected or unexpected with regard to outcome or severity of the event will be reported by the Sponsor as SUSAR to the competent authority, responsible ethics committee and investigators of the trial in line with the national regulations in effect (German drug law [AMG] and GCP-V $\S 13)$.

\section{Data Management and data quality assurance:}

Accurate and reliable data collection will be assured by verification and cross-check of the eCRF against the investigator's records by the study monitor (source document verification), and the maintenance of a drug-dispensing log by the investigator. Data for this study will be recorded via eCRF by the site from the source documents. Data are reviewed and checked for omissions, apparent errors, and values requiring further clarifications using computerized (automatic) and/or manual procedures. Data queries requiring clarification are communicated to the site for resolution via the CRO. Only authorized personnel will make corrections to the clinical database and an audit trail will document all corrections.

Information flow: Protocol amendments are submitted to the competent authorities and ethics committees for approval according to local legislation. Only after regulatory approval changes to the protocol are communicated to all sites via Mail or newsletter. Important safety information (e.g. SUSARS) are communicated directly to the participating investigator in parallel to reporting such information to the competent authorities. 
An overview of all study procedures is presented in table 3.

Treatment arms A and D: standard of care single agent or doublet chemotherapy.

Arm A: $n a b$-paclitaxel $100 \mathrm{mg} / \mathrm{m}^{2}$ on days $\mathrm{d} 1$ and $\mathrm{d} 8$ and carboplatin area under the curve (AUC) 5 on day 1 , every 3 weeks up to four cycles.

Arm D: Gemcitabine $1000 \mathrm{mg} / \mathrm{m}^{2}$ on days $\mathrm{d} 1$ and $\mathrm{d} 8$, given every 3 weeks, or vinorelbine $30 \mathrm{mg} / \mathrm{m}^{2}$ on days d1 and d8 every 3 weeks up to four cycles.

Treatment arms B and C: 2 cycles of single agent or doublet chemotherapy followed by durvalumab

Arm B: Two cycles of nab-paclitaxel $100 \mathrm{mg} / \mathrm{m}^{2}$ on days $\mathrm{d} 1$ and $\mathrm{d} 8$ and carboplatin area under the curve (AUC) 5 on day 1, every 3 weeks followed by durvalumab 1125 mg every 3 weeks for two cycles followed by maintenance with durvalumab $1500 \mathrm{mg}$ every 4 weeks.

Arm C: Two cycles of gemcitabine $1000 \mathrm{mg} / \mathrm{m}^{2}$ on days $\mathrm{d} 1$ and $\mathrm{d} 8$, given every 3 weeks or vinorelbine 30 $\mathrm{mg} / \mathrm{m}^{2}$ on days $\mathrm{d} 1$ and d8 every 3 weeks up to four cycles followed by durvalumab $1125 \mathrm{mg}$ every 3 weeks for two cycles followed by maintenance with durvalumab $1500 \mathrm{mg}$ every 4 weeks.

\section{$\underline{\text { Tissue and blood collection for exploratory endpoints }}$}

\section{Tissue collection}

For each patient a FFPE tumor tissue block (archival or recent) or a minimum of ten unstained slides of tumor sample (2-3 $\mu \mathrm{m}$ sections; slices must be recent and collected on slides provided by the sponsor) must be available for biomarker (PD-L1) evaluation as stated in the inclusion criteria. Biopsy should be excisional, incisional or core-needle. Fine-needle aspiration is insufficient. Tumor PD-L1 expression is measured by an immunohistochemistry assay using SP263 antibody. If a re-biopsy upon tumor progression under study treatment is performed, submission of this tumor material is highly valued.

\section{Blood collection}

Participation of patients in the biomarker program is voluntary and must be documented in the informed consent form. The time points for blood sampling are before start of any treatment at baseline and after two cycles of chemotherapy, as well as after 20 weeks of study participation for all patients with stable disease or tumor regression and at the time point of detection of tumor growth in patients with disease progression. In arms B and C, blood is additionally collected after the first cycle of durvalumab.

\section{Study endpoints}

\section{Primary endpoint}

The primary endpoint is the rate of treatment related grade III/IV adverse events (CTCAE V4.03). It will be calculated taking into account patients who have received at least one dose of study medication. 
Secondary endpoints

Secondary endpoints will be:

- Overall response rate (ORR) according to RECIST 1.1 criteria

- Progression-free survival (PFS) will be calculated from the date of subject randomization until the date of confirmed PD or death from any cause; if no event is observed (e.g. lost to follow-up) PFS is censored at the time of last tumor assessment

- Overall survival (OS) will be calculated from the date of subject randomization until the date of death from any cause; if no event is observed (e.g. lost to follow-up) OS is censored at the day of last subject contact

- AEs/SAEs according to CTCAE 4.03

- Health-related quality of life (HR-QoL) assessment using Functional Assessment of Cancer Therapy Lung (FACT-L) questionnaire, a standard instrument to determine QoL including lung specific domains (23)

\section{Exploratory endpoints}

\section{Exploratory analysis on tissue samples}

Patients will provide a tumor tissue sample at screening to determine PD-L1 expression level. This assessment will be centralized and performed by an immunohistochemistry assay using SP263 antibody. The results will be used to correlate PD-L1 staining intensity (proportion of positive tumor and immune cells) with durvalumab efficacy.

\section{Exploratory analysis on blood samples}

Blood samples that are collected at different time points will be used to characterize the immune response and investigate biological processes before, during and after the administration of the treatment. Flow cytometric (FCM) analysis will be used to characterize the immune response and the biological processes before, during and after the administration of the treatment. Whole blood samples will be analysed with this modality with respect to changes in T-cell composition. Abundances of immunostimulatory cytokines will be quantified by measuring serum pro- and anti-inflammatory cytokines. Analysis of mutational load on cfDNA will be performed.

\section{Statistical analysis}

The primary safety endpoint for the study is the occurrence of CTCAE grade III/IV toxicities assessed from the first dose to 90 days after the last dose of durvalumab. This is also the primary study endpoint on which the sample size calculation is based. According to the results presented at ASCO 2015 by Rizvi it is assumed that the probability for a CTC grade III/IV toxicity for patients from the pooled experimental arms $B+C$ receiving durvalumab amounts to $P_{B+C}=0.18$ (24). Based on reported data of selected treatment related adverse events (combination chemotherapy nab-paclitaxel/carboplatin (25), mono-chemotherapy 
gemcitabine/vinorelbine (7)) it is furthermore assumed that the rate of patients with a CTC grade III/IV toxicity in the pooled control arms $A+D$ receiving chemotherapy only amounts to $P_{A+D}=0.35$. With the planned number of patients of $\mathrm{N}=200$, the assumed difference between these two groups can be detected using a Chi-square test at a two-sided significance level of $\alpha=10 \%$ with a probability of $1-\beta=0.80$, also taking into account a dropout rate of $15 \%$. Sample size calculation was performed using ADDPLAN v6.1.

It should be noted that the study is not powered to detect significant differences with regard to the efficacy endpoints, since its primary aim is to assess safety and tolerability. Hence, no confirmatory evidence can be drawn from the efficacy evaluation. Accordingly, all p-values for efficacy outcomes are only to be interpreted descriptively and no adjustment for multiple testing will be done.

The null hypothesis for the primary (safety) endpoint of the trial is defined as $\mathrm{H}_{0}: \mathrm{P}_{B+C}=\mathrm{P}_{A+D}$ (i.e., the rate of patients with a CTC grade III/IV toxicity is equal in the pooled experimental arms $B+C$ and the pooled control arms $A+D$ ), which is tested against its alternative $H_{1}: P_{B+C} \neq P_{A+D}$ (i.e., there is a difference between the pooled experimental arms $B+C$ and the pooled control arms $A+D$ with regard to the rate of patients with a CTC grade III/IV toxicity). These hypotheses will be assessed at a two-sided significance level of $a=0.1$ using a Mantel-Haenszel Chi-square test adjusting for the stratum "adopted combination/not prone to combination". Missing data for the primary outcome variable will be replaced by using multiple imputation (26). The analysis of the primary endpoint will be based on the Safety Population comprising all patients enrolled who received at least one dose of study medication.

Secondary endpoints will be analyzed descriptively. The analysis of PFS will be performed analogously to the analysis of OS by calculating one-year and two-year rates, median times per group, conducting a stratified log rank test, calculating Kaplan Meier curves and estimating the hazard ratio using a Cox regression adjusting for the stratum "adopted combination/not prone to combination". Other secondary endpoints will be analyzed descriptively by tabulating the measures of the empirical distributions. Subgroup analyses according to PD-L1 expression will be performed. A detailed methodology for the statistical analysis will be described in the statistical analysis plan (SAP), which will be finalized before data base lock. Statistical analysis will be done using SAS v9.4 or higher (SAS Institute, Cary, NC).

\section{Discussion}

Lung cancer is the most common cause of cancer related death worldwide and it is predominantly a disease of the elderly, with about $50 \%$ of patients diagnosed aged 70 years or older and with about $14 \%$ of these being older than 80 years (2). Due to the fact that lung cancer is mostly diagnosed at an advanced stage, prognosis is very poor.

Chemotherapy is effective in elderly NSCLC patients. However, they might experience treatment toxicity and deterioration due to side effects. The Elderly Selection on Geriatric Index Assessment (ESOGIA) trial was the first prospective study to investigate comprehensive geriatric assessment (CGA) incorporation into cancer treatment decisions and its impact on survival outcomes (27). The study randomly assigned 192 stage IV NSCLC patients with a median age of 77 years to a standard arm or a CGA arm, where patients

Page $11 / 28$ 
received either one of two chemotherapy regimens or best supportive care (BSC) based on performance status (PS) and age or on the CGA evaluation, respectively. Importantly, the treatment allocation based on CGA reduced treatment toxicities and the number of toxicity-related treatment failures, although it was not able to improve treatment failure-free survival or OS. This trial for the first time demonstrated the feasibility of incorporating CGA in a multicenter clinical trial setting and that CGA-based treatment is associated with decreased toxicity in elderly NSCLC patients. In clinical practice, however, the implementation of CGA has been difficult because it is rather time- and resource-consuming. Consequently, alternative pre-therapy risk assessment tools have been developed to predict chemotherapy toxicity, the CRASH and CARG scores being the two most promising tools for assigning patients to varying chemotherapy intensities based on pre-therapy risk assessment.

In the DURATION trial, the CARG toxicity prediction tool will be used to guide treatment intensity with the intention to improve outcomes of elderly and frail patients. The CARG score has been developed to stratify patients and identify those at higher risk for chemotherapy toxicity (10). It consists of eleven questions, including five geriatric assessments questions and six clinical questions concerning items retrieved from everyday practice. The CARG score was validated in lung cancer, showing its value in better distinguishing the risks of chemotherapy toxicity in older patients compared to the Karnofsky performance status (KPS) (28). Its value in treating and predicting mortality in elderly patients with cancer is now broadly accepted. Minor modifications of the CARG score in the DURATION trial include the removal of the default scoring items "polychemotherapy" and standard dose as well as the items "GI" or "GU cancer", which do not apply to this study. The predictive properties of the CARG score remain unchanged.

According to this modified CARG toxicity tool, patients in the DURATION trial will be classified as "fit" or as "less fit" with regard to receiving a platinum-based combination chemotherapy. "Less fit" patients will be treated with a single-agent chemotherapy of either vinorelbine or gemcitabine. Both single-agent chemotherapy regimens were established as the standard of care over best supportive care for $1^{\text {st }}$-line therapy of advanced NSCLC patients aged 70 years or older $(5,29)$. Patients that are stratified as "fit" will receive treatment according to current ESMO guidelines for advanced NSCLC that recommend platinumbased combination chemotherapy for patients aged $>70$ years with PS 0-2 and adequate organ function based on a recent systematic review (30). The combination chemotherapy applied in the DURATION trial consists of a combination of nab-paclitaxel with carboplatin as inferred from clinical trials and retrospective analyses that demonstrated superiority of carboplatin/nab-paclitaxel over carboplatin/paclitaxel with respect to efficacy and safety in elderly patients $(25,31)$. Both patient groups treated with either single-agent or doublet chemotherapy will be subjected to randomization for treatment with the PD-L1 inhibitor durvalumab.

Based on promising results from clinical trials, immuno-oncology agents such as PD-1 - or PD-L1 inhibitors have found their way into frequent clinical use, even in the $1^{\text {st }}$-line setting and have revolutionized the treatment landscape of NSCLC (32). However, due to underrepresentation of older patients in large trials that led to approval of checkpoint inhibitors, all available efficacy and safety data for this patient group is derived from subgroup analyses. Such analyses of $2^{\text {nd }}$-line trials revealed no 
differences in response rates and survival between patients aged less or more than 65 years $(12,14,18,33,34)$. Similarly, KEYNOTE-024, a $1^{\text {st }}$-line clinical trial comparing pembrolizumab with combination chemotherapy in advanced NSCLC patients with PD-L1 expression $>50 \%$, indicated no differences in the beneficial effect of pembrolizumab when comparing patients aged $<65$ years and >65 years) (16). Of note, no differences regarding toxicities between age groups were observed (14). However, to date no data from randomized phase III trials assessing the efficacy of PD-1/PD-L1 targeting agents in elderly patients with advanced NSCLC are available.

In addition, addressing immuno-oncology agents specifically in older patients is of particular interest as a phenomenon called immunosenescence has to be considered. This age-related decline in the immune system includes reductions in B- and T-cell proliferation and function, quantitative differences in cellular subsets, functional impairment, and qualitative changes in APCs and an accumulation of regulatory $T$ cells - processes that eventually could be associated with impaired immune response to pathogens and tumor cells (18).

Considering the growing number of immune checkpoint inhibitors that are available for the treatment of NSCLC patients, it is also important to learn about potential differences between PD-1 and PD-L1 targeting agents. Although each drug has shown activity in NSCLC, comparing these agents in terms of efficacy and toxicity is subject of current research. A recent systematic review of clinical trials that tested both PD-1 and PD-L1 antibodies did not find significant differences between the two types of checkpoint inhibitors regarding the reported response rates and toxicity profiles (35). The most notable difference was observed regarding grade III/IV immune-mediated pneumonitis that was slightly higher with PD-1 inhibitors compared with PD-L1 inhibitors. This could possibly be explained by the fact that anti-PD-L1 antibodies still allow for the interaction of PD-1 with its other ligand PD-L2, thus resulting in a weaker blockade of the negative inhibitory signal and reduced autoimmunity (35). The development of autoimmune pneumonitis has to be carefully monitored in NSCLC patients as this has led to a few treatment-related deaths in earlyphase studies of PD-1 targeting agents $(13,36,37)$ and patients with lung cancer are more vulnerable to toxicities given the older age of the patient population and the presence of comorbid conditions. Given the putatively lower risk of developing autoimmune-mediated toxicities with PD-L1 targeting agents, the use of durvalumab, a selective, high-affinity, human IgG1 monoclonal anti-PD-L1 antibody (38-40), is expected to be more suitable for treatment of a more vulnerable patient group such as old or frail NSCLC patients that are included in the DURATION trial. Encouraging antitumor activity of durvalumab has already been shown in an early-phase clinical study involving multiple advanced solid tumors, including NSCLC, and recently it has been approved for patients with locally advanced NSCLC after chemoradiotherapy $(38,41)$.

In the DURATION trial, checkpoint inhibition by durvalumab is combined sequentially with cytotoxic chemotherapy. It is thought that modulation of the immune response through PD-1 inhibition may be enhanced by the potential immunogenic effects of cytotoxic chemotherapy, e.g. by increasing the potential for antigen cross-presentation by dendritic cells after the destruction of tumor cells, inhibiting myeloidderived suppressor cells, increasing the ratio of cytotoxic lymphocytes to regulatory $T$ cells, and blocking the STAT6 pathway to enhance dendritic-cell activity $(42-45)$. Thus, the two cycles of induction 
chemotherapy that are applied in the DURATION trial are expected to lead to a prompt disease stabilizing effect, which can be efficaciously extended by a consecutive PD-L1 immunotherapy with durvalumab.

Current treatment guidelines recommend the use of immunotherapy alone or in combination with CT also for older lung cancer patients with adequate PS ECOG(46). Considering the underrepresentation of older and frail patients in main pivotal trials that led to the approval of these new treatment modalities, there's a lack of data about safety and efficacy in this group. Aging processes, comorbidities and undetected frailty could affect treatment tolerance in face of a poor clinical benefit. The DURATION trial will help to close the current gap in knowledge about safety and tolerability of checkpoint inhibitors in elderly and frail lung cancer patients, an important cohort that has been underrepresented in clinical trials for too long.

\section{Trial Status}

Patient accrual started in December 2017 with protocol version 5.0 and is currently ongoing according to the protocol version number 7. Two Amendments were necessary, due to annual updated IB of Durvalumab, leading to protocol version 6.0 (approval 24.05.2018) and version 7.0 (approval 27.06.2019). At present, 30 centers are participating in this study. The approximate end of recruitment will be in December 2020.

\section{List Of Abbreviations}

$A E$

AIO

APC

ASCO

AUC

BSC

CARG

$\mathrm{CCl}$

$C D$

cfDNA

CGA

CRASH
Adverse event

Arbeitsgemeinschaft Internistische Onkologie

Antigen presenting cells

American society of clinical oncology

Area under the curve

Best supportive care

Cancer and aging research group

Charlson comorbidity index

Cluster of differentiation

cell-free Deoxyribose nucleic acid

comprehensive geriatric assessment

Chemotherapy Risk Assessment Scale for High-Age Patients 


\begin{tabular}{|c|c|}
\hline CT & Computed tomography \\
\hline CTCAE & Common terminology criteria for adverse events \\
\hline ECOG & Eastern Cooperative Oncology Group \\
\hline EGFR & Epidermal growth factor receptor \\
\hline EML4ALK & Echinoderm microtubule-associated protein-like 4 anaplastic lymphoma kinase \\
\hline ESMO & European Society for Medical Oncology \\
\hline FACS & Fluorescence activated cell sorter \\
\hline FFPE & Formalin-fixed, paraffin-embedded \\
\hline GI & Gastrointestinal \\
\hline GU & Genitourinary \\
\hline G8 & Geriatric 8 \\
\hline HBV & Hepatitis B virus \\
\hline $\mathrm{HCV}$ & Hepatitis C virus \\
\hline HR-QoL & Health related quality of life \\
\hline $\mathrm{IHC}$ & Immunohistochemistry \\
\hline IMP & Investigational medical product \\
\hline irRECIST & Immune-related response evaluation criteria in solid tumors \\
\hline MRI & Magnetic resonance imaging \\
\hline 6MWT & Six minute walk test \\
\hline NSCLC & Non-small cell lung cancer \\
\hline ORR & Objective response rate \\
\hline OS & Overall survival \\
\hline PD-1 & Programmed death-1 \\
\hline PD-L1 & Programmed death-ligand 1 \\
\hline
\end{tabular}


PFS Progression-free survival

RECIST Response evaluation criteria in solid tumors

SAE Severe adverse event

QoL Quality of life

\section{Declarations}

\section{Ethics approval and consent to participate}

Central ethical approval has been confirmed from the Ethics Committee of the Medical Faculty of the University of Heidelberg 24.10.2017 (AFmu-681/2016) and we will not begin recruiting at other centres in the trial until local ethical approval has been obtained.

Written informed consent is obtained from all participants. Additionally, the Paul Ehrlich Institute (competent authority for approval of clinical trials using medicinal products for human use in Germany) approved the study on 03.07.2017

\section{Consent for publication}

Not applicable - as no individual patient data are contained in this manuscript.

\section{Availability of data and material}

Not applicable - as no primary data are contained, generated or analysed.

\section{Competing interests}

The DURATION trial received funding from AstraZeneca and Celgene. AstraZeneca and Celgene have not been involved in the study design, and has no role in data collection, management, data analysis and interpretation, or in the decision to submit this protocol for publication.

All authors declare that there are no competing conflicts of interest.

\section{Funding}

DURATION is an investigator-initiated trial that is supported by the non-profit AIO-Studien-gGmbH (Berlin, Germany) as legal sponsor. Funding and drugs for this trial are provided by AstraZeneca and Celgene.

\section{Authors' contributions}

JK1, MT developed the study idea, wrote the protocol and coordinated funding, regulatory permission and approval processes. JK1 is lead investigator of this study. FB is substitute lead investigator. JK1 wrote the manuscript in consultation with $\mathrm{MB}$. AH developed preclinical, translational and biomarker exploratory 
projects, derived from DURATION. JK2 calculated (bio-) statistical models for sample size, study hypothesis, and endpoint determinations. FL and AS performs tissue-based biomarker evaluation strategies and will perform all respective analysis. MM is representative of the AlO-Studien-gGmbH (KunoFischer-Str. 8, 14,057 Berlin, Germany, info@aio-studien-ggmbh.de) and substantially contributed to conception, design and preparation of study protocol. The AlO-Studien-gGmbH is sponsor of the study and responsible for study management, logistics and counselled in study design.

\section{Acknowledgements}

The authors would like to thank the members of the Protocol Board and the Data Safety and Monitoring Board. They would also like to thank Inn Chung for comments on the manuscript.

This trial is conducted by the Young Medical Oncologists (YMO) Group in cooperation with the Thoracic Oncology Working Group of the Arbeitsgemeinschaft Internistische Onkologie (AIO) within the German Cancer Society.

\section{Authors' information (optional)}

not provided

\section{Availability of data and materials}

Not applicable.

\section{References}

1. Bray F, Ferlay J, Soerjomataram I, Siegel RL, Torre LA, Jemal A. Global cancer statistics 2018: GLOBOCAN estimates of incidence and mortality worldwide for 36 cancers in 185 countries. CA Cancer J Clin. 2018 Nov;68(6):394-424.

2. Owonikoko TK, Ragin CC, Belani CP, Oton AB, Gooding WE, Taioli E, et al. Lung cancer in elderly patients: An analysis of the surveillance, epidemiology, and end results database. J Clin Oncol. 2007 Dec 10;25(35):5570-7.

3. Tas F, Ciftci R, Kilic L, Karabulut S. Age is a prognostic factor affecting survival in lung cancer patients. Oncol Lett. 2013 Nov;6(5):1507-13.

4. Jackman DM, Zhang Y, Dalby C, Nguyen T, Nagle J, Lydon CA, et al. Cost and Survival Analysis Before and After Implementation of Dana-Farber Clinical Pathways for Patients With Stage IV Non-SmallCell Lung Cancer. J Oncol Pract. 2017 Apr;13(4):e346-52.

5. Gridelli C, Perrone F, Gallo C, Cigolari S, Rossi A, Piantedosi F, et al. Chemotherapy for elderly patients with advanced non-small-cell lung cancer: The multicenter Italian lung cancer in the elderly study (MILES) phase III randomized trial. J Natl Cancer Inst. 2003 Mar 5;95(5):362-72.

6. Langer C. MTE 22.02 Treatment Options in Advanced Non-Small Cell Lung Cancer (NSCLC) in the Elderly: An Evolving Landscape. J Thorac Oncol. 2017 Nov;12(11):S1651-2. 
7. Quoix E, Zalcman G, Oster JP, Westeel V, Pichon E, Lavolé A, et al. Carboplatin and weekly paclitaxel doublet chemotherapy compared with monotherapy in elderly patients with advanced non-small-cell lung cancer: IFCT-0501 randomised, phase 3 trial. Lancet. 2011 Sep 17;378(9796):1079-88.

8. Wildiers H, Heeren P, Puts M, Topinkova E, Janssen-Heijnen MLG, Extermann M, et al. International society of geriatric oncology consensus on geriatric assessment in older patients with cancer. Vol. 32, Journal of Clinical Oncology. American Society of Clinical Oncology; 2014. p. 2595-603.

9. Extermann M, Boler I, Reich RR, Lyman GH, Brown RH, Defelice J, et al. Predicting the risk of chemotherapy toxicity in older patients: The chemotherapy risk assessment scale for high-age patients (CRASH) score. Cancer. 2012 Jul 1;118(13):3377-86.

10. Hurria A, Togawa K, Mohile SG, Owusu C, Klepin HD, Gross CP, et al. Predicting chemotherapy toxicity in older adults with cancer: A prospective multicenter study. J Clin Oncol. 2011;29(25):3457-65.

11. Almodovar T, Teixeira E, Barroso A, Soares M, Queiroga HJ, Cavaco-Silva J, et al. Elderly patients with advanced NSCLC: The value of geriatric evaluation and the feasibility of CGA alternatives in predicting chemotherapy toxicity. Vol. 25, Pulmonology. Elsevier Espana S.L.U; 2019. p. 40-50.

12. Borghaei H, Paz-Ares L, Horn L, Spigel DR, Steins M, Ready NE, et al. Nivolumab versus docetaxel in advanced nonsquamous non-small-cell lung cancer. N Engl J Med. 2015 Oct 22;373(17):1627-39.

13. Garon EB, Rizvi NA, Hui R, Leighl N, Balmanoukian AS, Eder JP, et al. Pembrolizumab for the treatment of non-small-cell lung cancer. N Engl J Med. 2015 May 21;372(21):2018-28.

14. Rittmeyer A, Barlesi F, Waterkamp D, Park K, Ciardiello F, von Pawel J, et al. Atezolizumab versus docetaxel in patients with previously treated non-small-cell lung cancer (OAK): a phase 3, open-label, multicentre randomised controlled trial. Lancet. 2017 Jan 21;389(10066):255-65.

15. Socinski MA, Jotte RM, Cappuzzo F, Orlandi F, Stroyakovskiy D, Nogami N, et al. Atezolizumab for firstline treatment of metastatic nonsquamous NSCLC. N Engl J Med. 2018 Jun 14;378(24):2288-301.

16. Reck M, Rodriguez-Abreu D, Robinson AG, Hui R, Csöszi T, Fülöp A, et al. Pembrolizumab versus Chemotherapy for PD-L1-Positive Non-Small-Cell Lung Cancer. N Engl J Med. 2016 Nov 10;375(19):1823-33.

17. Gandhi L, Rodríguez-Abreu D, Gadgeel S, Esteban E, Felip E, De Angelis F, et al. Pembrolizumab plus chemotherapy in metastatic non-small-cell lung cancer. N Engl J Med. 2018 May 31;378(22):207892.

18. Ferrara R, Mezquita L, Auclin E, Chaput N, Besse B. Immunosenescence and immunecheckpoint inhibitors in non-small cell lung cancer patients: Does age really matter? Cancer Treatment Reviews. 2017.

19. Daste A, Domblides C, Gross-goupil M, Chakiba C, Quivy A, Cochin V, et al. Immune checkpoint inhibitors and elderly people: A review. Vol. 82, European Journal of Cancer. Elsevier Ltd; 2017. p. 155-66.

20. Champiat S, Dercle L, Ammari S, Massard C, Hollebecque A, Postel-Vinay S, et al. Hyperprogressive disease is a new pattern of progression in cancer patients treated by anti-PD-1/PD-L1. Clin Cancer Res. 2017 Apr 15;23(8):1920-8. 
21. Fulop T, Kotb R, Fortin CF, Pawelec G, De Angelis F, Larbi A. Potential role of immunosenescence in cancer development. In: Annals of the New York Academy of Sciences. Blackwell Publishing Inc.; 2010. p. 158-65.

22. Hurria A, Gupta S, Zauderer M, Zuckerman EL, Cohen HJ, Muss H, et al. Developing a cancer-specific geriatric assessment: A feasibility study. Vol. 104, Cancer. 2005. p. 1998-2005.

23. Cella DF, Tulsky DS, Gray G, Sarafian B, Linn E, Bonomi A, et al. The Functional Assessment of Cancer Therapy scale: development and validation of the general measure. J Clin Oncol. 1993 Mar;11(3):570-9.

24. Rizvi NA, Brahmer JR, Ou S-HI, Segal NH, Khleif S, Hwu W-J, et al. Safety and clinical activity of MEDI4736, an anti-programmed cell death-ligand 1 (PD-L1) antibody, in patients with non-small cell lung cancer (NSCLC). J Clin Oncol. 2015 May 20;33(15_suppl):8032-8032.

25. Socinski MA, Bondarenko I, Karaseva NA, Makhson AM, Vynnychenko I, Okamoto I, et al. Weekly nabpaclitaxel in combination with carboplatin versus solvent-based paclitaxel plus carboplatin as firstline therapy in patients with advanced non-small-cell lung cancer: Final results of a phase III trial. J Clin Oncol. 2012 Jun 10;30(17):2055-62.

26. Demirtas H. Flexible Imputation of Missing Data. J Stat Softw. 2018;85(Book Review 4).

27. Corre R, Greillier L, Le Caër H, Audigier-Valette C, Baize N, Bérard H, et al. Use of a comprehensive geriatric assessment for the management of elderly patients with advanced non-small cell lung cancer: The Phase III randomized ESOGIA-GFPC-GECP 08-02 Study. J Clin Oncol. 2016 May 1;34(13):1476-83.

28. Nie X, Liu D, Li Q, Bai C. Predicting chemotherapy toxicity in older adults with lung cancer. J Geriatr Oncol. 2013 Oct;4(4):334-9.

29. Kudoh S, Takeda K, Nakagawa K, Takada M, Katakami N, Matsui K, et al. Phase III study of docetaxel compared with vinorelbine in elderly patients with advanced non-small-cell lung cancer: Results of the West Japan Thoracic Oncology Group trial (WJTOG 9904). J Clin Oncol. 2006 Aug 1;24(22):3657-63.

30. Santos FN, de Castria TB, Cruz MRS, Riera R. Chemotherapy for advanced non-small cell lung cancer in the elderly population. Vol. 2015, Cochrane Database of Systematic Reviews. John Wiley and Sons Ltd; 2015.

31. Gridelli C, Chen T, Ko A, O’Brien ME, Ong TJ, Socinski MA, et al. Nab-paclitaxel/carboplatin in elderly patients with advanced squamous non-small cell lung cancer: A retrospective analysis of a phase iii trial. Drug Des Devel Ther. 2018 May 24;12:1445-51.

32. Lopes G, Wu Y-L, Kudaba I, Kowalski D, Cho BC, Castro G, et al. Pembrolizumab (pembro) versus platinum-based chemotherapy (chemo) as first-line therapy for advanced/metastatic NSCLC with a PD-L1 tumor proportion score (TPS) $\geq 1 \%$ : Open-label, phase 3 KEYNOTE-042 study. J Clin Oncol. 2018 Jun 20;36(18_suppl):LBA4-LBA4.

33. Sawahashi M, Kishiyama Y, Morimoto A, Nishikawa D, Tanno M. Coordinated multipoint transmission/reception techniques for LTE-advanced. IEEE Wirel Commun. 2010 Jun;17(3):26-34. 
34. Herbst RS, Baas P, Kim DW, Felip E, Pérez-Gracia JL, Han JY, et al. Pembrolizumab versus docetaxel for previously treated, PD-L1-positive, advanced non-small-cell lung cancer (KEYNOTE-010): A randomised controlled trial. Lancet. 2016 Apr 9;387(10027):1540-50.

35. Pillai RN, Behera M, Owonikoko TK, Kamphorst AO, Pakkala S, Belani CP, et al. Comparison of the toxicity profile of PD-1 versus PD-L1 inhibitors in non-small cell lung cancer: A systematic analysis of the literature. Vol. 124, Cancer. John Wiley and Sons Inc.; 2018. p. 271-7.

36. Gettinger SN, Horn L, Gandhi L, Spigel DR, Antonia SJ, Rizvi NA, et al. Overall survival and long-term safety of nivolumab (anti-programmed death 1 antibody, BMS-936558, ONO-4538) in patients with previously treated advanced non-small-cell lung cancer. J Clin Oncol. 2015 Jun 20;33(18):2004-12.

37. Topalian SL, Hodi FS, Brahmer JR, Gettinger SN, Smith DC, McDermott DF, et al. Safety, activity, and immune correlates of anti-PD-1 antibody in cancer. N Engl J Med. 2012 Jun 28;366(26):2443-54.

38. Pardoll DM. The blockade of immune checkpoints in cancer immunotherapy. Vol. 12, Nature Reviews Cancer. 2012. p. 252-64.

39. Postow MA, Callahan MK, Wolchok JD. Immune checkpoint blockade in cancer therapy. Vol. 33, Journal of Clinical Oncology. American Society of Clinical Oncology; 2015. p. 1974-82.

40. Stewart R, Morrow M, Hammond SA, Mulgrew K, Marcus D, Poon E, et al. Identification and characterization of MEDI4736, an antagonistic anti-PD-L1 monoclonal antibody. Cancer Immunol Res. 2015 Sep 1;3(9):1052-62.

41. Antonia SJ, Villegas A, Daniel D, Vicente D, Murakami S, Hui R, et al. Durvalumab after chemoradiotherapy in stage III non-small-cell lung cancer. N Engl J Med. 2017;

42. Bracci L, Schiavoni G, Sistigu A, Belardelli F. Immune-based mechanisms of cytotoxic chemotherapy: Implications for the design of novel and rationale-based combined treatments against cancer. Vol. 21, Cell Death and Differentiation. 2014. p. 15-25.

43. Wang Z, Till B, Gao Q. Chemotherapeutic agent-mediated elimination of myeloid-derived suppressor cells. Oncoimmunology. 2017;6(7).

44. Roselli M, Cereda V, Giovanna di Bari M, Formica V, Spila A, Jochems C, et al. Effects of conventional therapeutic interventions on the number and function of regulatory $T$ cells. Oncoimmunology. 2013;2(10).

45. Lesterhuis WJ, Punt CJA, Hato S V., Eleveld-Trancikova D, Jansen BJH, Nierkens S, et al. Platinumbased drugs disrupt STAT6-mediated suppression of immune responses against cancer in humans and mice. J Clin Invest. 2011;121(8):3100-8.

46. Planchard D, Popat S, Kerr K, Novello S, Smit EF, Faivre-Finn C, et al. Metastatic non-small cell lung cancer: ESMO Clinical Practice Guidelines for diagnosis, treatment and follow-up. Ann Oncol. 2018;29:iv192-237.

\section{Tables}

Table 1 : Complete list of inclusion / exclusion criteria 


\section{Inclusion criteria}

1. Written informed consent and any locally-required authorization (EU Data Privacy Directive in the EU) obtained from the subject prior to performing any protocol-related procedures, including screening evaluations.

2. $\quad$ Age $\geq 70$ years at time of study entry and / or Charlson-Comorbidity-Index (CCI) $>1$ and / or Performance status PS $>1$

3. - Histologically confirmed diagnosis of metastatic NSCLC and no targetable molecular alterations (EGFR WT; ALK transl-)

4. $\quad$ Patients with measurable disease according to Response Evaluation Criteria in Solid Tumors (RECIST 1.1)

5. - A formalin fixed, paraffin-embedded (FFPE) tumor tissue block or a minimum of 10 unstained slides of tumor sample

6. - No prior chemotherapy or any other systemic therapy for metastatic NSCLC. Patients who received prior platinum-containing adjuvant, neoadjuvant, or definitive chemoradiation for locally advanced disease are eligible, provided that progression has occurred $>6$ months from last therapy.

7. Prior radiotherapy and surgery are allowed if completed 4 weeks prior to start of treatment and patient recovered from toxic effects or associated adverse events.

8. Adequate blood count, liver-enzymes, and renal function

- Haemoglobin $\geq 9.0 \mathrm{~g} / \mathrm{dl}$

- Absolute neutrophil count (ANC) $\geq 1.5 \times 10^{9} / \mathrm{L}\left(>100\right.$ per $\left.\mathrm{mm}^{3}\right)$

- platelet count $\geq 100 \times 10^{9} / \mathrm{L}\left(>100,00\right.$ per $\left.\mathrm{mm}^{3}\right)$

- Serum bilirubin $\leq 1.5 \mathrm{x}$ ULN. This will not apply to subjects with confirmed Gilbert's syndrome (persistent or recurrent hyperbilirubinemia that is predominantly unconjugated in the absence of hemolysis or hepatic pathology), who will be allowed only in consultation with their physician. 
- AST (SGOT)/ALT (SGPT) $\leq 2.5 \mathrm{x}$ institutional upper limit of normal unless liver metastases are present, in which case it must be $\leq 5 \mathrm{x}$ ULN

- Serum creatinine CL>40 mL/min by the Cockcroft-Gault formula (Cockcroft and Gault 1976) or by 24hour urine collection for determination of creatinine clearance

- Subject is willing and able to comply with the protocol for the duration of the study including undergoing treatment and scheduled visits, examinations including follow up and appropriate contraception.

\section{Exclusion criteria}

- Mixed small-cell lung cancer and NSCLC histology

- Mean QT interval corrected for heart rate (QTc) $\geq 470$ ms calculated from 3 electrocardiograms (ECGs) using Fredericia's correction

- History of another primary malignancy except local prostate cancer without need for systemic treatment (e.g. active surveillance, operation without need for adjuvant treatment) and malignancies treated with curative intent and with no known active disease $>2$ years before the first dose of study drug and of low potential risk for recurrence - adequately treated non-melanoma skin cancer or lentigo maligna without evidence of disease - adequately treated carcinoma in situ without evidence of disease (e.g. cervical cancer in situ)

- Pre-existing peripheral neuropathy of Grade $\geq 2$

- Brain metastasis or spinal cord compression unless asymptomatic or treated and stable off steroids and anticonvulsants for at least 1 month prior to study treatment.

- Active or prior documented autoimmune disease within the past 2 years. NOTE: Subjects with vitiligo, Grave's disease, or psoriasis not requiring systemic treatment (within the past 2years) are not excluded. 
- Active or prior documented inflammatory bowel disease (e.g., Crohn's disease, ulcerative colitis)

- History of primary immunodeficiency

- History of allogeneic organ transplant

- History of hypersensitivity to durvalumab or any excipient

- History of hypersensitivity to any of the comparator agents

- Medication that is known to interfere with any of the agents applied in the trial

- Uncontrolled intercurrent illness including, but not limited to, ongoing or active infection, symptomatic congestive heart failure, uncontrolled hypertension, unstable angina pectoris, cardiac arrhythmia, active peptic ulcer disease or gastritis, active bleeding diatheses including any subject known to have evidence of acute or chronic hepatitis B, hepatitis C or human immunodeficiency virus (HIV), or psychiatric illness/social situations that would limit compliance with study requirements or compromise the ability of the subject to give written informed consent

- Clinical diagnosis of active tuberculosis

- Receipt of live attenuated vaccination within 30 days prior to study entry or within 30 days of receiving durvalumab

- Male patients of reproductive potential who are not employing an effective method of birth control (failure rate of less than $1 \%$ per year)

- Any condition that, in the opinion of the investigator, would interfere with evaluation of study treatment or interpretation of patient safety or study results

- Participation in another clinical study with an investigational product during the last 30 days before inclusion

- Any previous treatment with a PD-1 or PD-L1 inhibitor, including durvalumab

- Current or prior use of immunosuppressive medication within 28 days before the first dose of durvalumab, with the exceptions of intranasal and inhaled corticosteroids or systemic corticosteroids at physiological doses, which are not to exceed $10 \mathrm{mg} /$ day of prednisone, or an equivalent corticosteroid 
- Receipt of the last dose of anti-cancer therapy (chemotherapy, immunotherapy, endocrine therapy, targeted therapy, biologic therapy, tumor embolization, monoclonal antibodies, other investigational agent) $\leq 21$ days prior to the first dose of study drug or $\leq 4$ half-lifes of the agent administered, whichever comes first.

- Previous enrollment or randomization in the present study.

- Involvement in the planning and/or conduct of the study(applies to both AstraZeneca staff and/or staff of sponsor and study site)

- Patient who might be dependent on the sponsor, site or the investigator

- Patient who has been incarcerated or involuntarily institutionalized by court order or by the authorities $\S$ 40 Abs. 1 S. 3 Nr. 4 AMG.

- Patients who are unable to consent because they do not understand the nature, significance and implications of the clinical trial and therefore cannot form a rational intention in the light of the facts [§ 40 Abs. 1 S. 3 Nr. 3a AMG].

Table 2: Modified CARG Risk score determination for treatment stratification. [Modified from Hurria et al. JCO 2011] 


\begin{tabular}{llc} 
Toxicity Factor / Question & Value/Response & Score \\
\hline Age $\geq 72$ years & $\geq 72$ & 2 \\
Hemoglobin & $<11 \mathrm{~g} / \mathrm{dL}$ (male) & 3 \\
& $<10 \mathrm{~g} / \mathrm{dL}$ (female) & 2 \\
Creatinine clearance & $<34 \mathrm{~mL} / \mathrm{min}$ & 3 \\
Hearing & Fair / worse & 1 \\
Number of falls in the past 6 months & 1 or more \\
Taking medications & with some help or unable \\
Walking 1 block (100 meters) & somewhat limited or limited a lot 2 \\
Decreased social activity because of physical/emotional health & limited at least sometimes & 1 \\
\hline
\end{tabular}

\section{Table 3: schedule of assessments}

\section{Schedule of assessments}




\begin{tabular}{|c|c|c|c|c|c|c|c|c|}
\hline \multirow{6}{*}{$\begin{array}{l}\text { Procedure / } \\
\text { Assessment }\end{array}$} & \multirow{6}{*}{$\begin{array}{c}\text { Screening } \\
-28 d \\
\text { before } \\
\text { C1D1 } \\
\text { Inclusion }\end{array}$} & & & & & Arm B \& C & All A & \\
\hline & & \multirow{2}{*}{\multicolumn{4}{|c|}{$\begin{array}{c}\text { Treatment (q3w) } \\
+/-2 d\end{array}$}} & \multirow{5}{*}{$\begin{array}{l}\text { Maintenance } \\
\qquad \begin{array}{r}(\mathrm{q} 4 \mathrm{w}) \\
+/-7 \mathrm{~d}\end{array} \\
\text { C5 - Cx }\end{array}$} & \multirow{5}{*}{$\begin{array}{c}\text { End of } \\
\text { Treatment }\end{array}$} & \multirow{5}{*}{$\begin{array}{l}\text { Follow- } \\
\qquad \begin{array}{l}\text { Up } \\
+/-7 d\end{array}\end{array}$} \\
\hline & & & & & & & & \\
\hline & & \multirow{2}{*}{\multicolumn{2}{|c|}{$\begin{array}{c}\text { C1 - C2 (Arm } \\
\text { B/C) } \\
\text { C1-C4 (Arm A/D) }\end{array}$}} & \multirow{3}{*}{$\begin{array}{c}\text { C3 (Arm } \\
\text { B/C) }\end{array}$} & \multirow{3}{*}{$\begin{array}{c}\text { C4 (Arm } \\
\text { B/C) }\end{array}$} & & & \\
\hline & & & & & & & & \\
\hline & & Day 1 & Day 8 & & & & & \\
\hline $\begin{array}{l}\text { Informed } \\
\text { consent, } \\
\text { eligibility } \\
\text { criteria, } \\
\text { demographics, } \\
\text { medical and } \\
\text { disease } \\
\text { history }\end{array}$ & $\mathrm{x}$ & & & & & & & \\
\hline $\begin{array}{l}\text { FFPE tumor } \\
\text { tissue (PD-L1) }\end{array}$ & $\mathrm{x}$ & & & & & & & \\
\hline $\begin{array}{l}\text { Vital signs, } \\
\text { physical }\end{array}$ & $\mathrm{x}$ & $\mathrm{x}$ & $\mathrm{x}$ & $\mathrm{x}$ & $\mathrm{x}$ & $\mathrm{x}$ & $\mathrm{x}$ & \\
\hline ECOG & $\mathrm{x}$ & & & $\mathrm{x}$ & & $\mathrm{x}$ & $\mathrm{x}$ & $\mathrm{x}^{\mathrm{a}}$ \\
\hline $\mathrm{AE} / \mathrm{SAE}$ & $\mathrm{x}$ & $\mathrm{x}$ & $\mathrm{x}$ & $\mathrm{x}$ & $\mathrm{x}$ & $\mathrm{x}$ & $\mathrm{x}$ & $\mathrm{x}$ \\
\hline $\begin{array}{l}\text { CT / MRI of } \\
\text { tumor lesions }\end{array}$ & $\mathrm{x}$ & & & $\mathrm{x}$ & & $x^{b}$ & & $\mathrm{x}^{\mathrm{C}}$ \\
\hline HR-QoL & $\mathrm{x}$ & & & $\mathrm{x}$ & & $x^{b}$ & $\mathrm{x}$ & $\mathrm{x}$ \\
\hline $\begin{array}{l}\text { Charlson } \\
\text { Comorbidity } \\
\text { Index }\end{array}$ & $\mathrm{x}$ & & & & & & & \\
\hline
\end{tabular}




\begin{tabular}{|c|c|c|c|c|c|c|c|c|}
\hline CARG-score & $\mathrm{x}$ & & & & & & & \\
\hline $\begin{array}{l}\text { Geriatric } \\
\text { assessments }\end{array}$ & $\mathrm{x}$ & & & & & $\mathrm{x}$ & $\mathrm{x}$ & $x^{d}$ \\
\hline $\begin{array}{l}\text { Biomarker } \\
\text { sample }\end{array}$ & & $\mathrm{x}^{\mathrm{e}}$ & & $\mathrm{x}$ & $x^{f}$ & $x^{g}$ & $\mathrm{x}$ & $\mathrm{x}^{\mathrm{a}}$ \\
\hline \multicolumn{9}{|l|}{ Treatments } \\
\hline Arm A & & CHT & CHT & $\mathrm{CHT}$ & $\mathrm{CHT}$ & & & \\
\hline Arm B & & $\mathrm{CHT}$ & $\mathrm{CHT}$ & Durvalumab & Durvalumab & Durvalumab & & \\
\hline Arm C & & CHT & CHT & Durvalumab & Durvalumab & Durvalumab & & \\
\hline Arm D & & CHT & CHT & CHT & CHT & & & \\
\hline
\end{tabular}

a: at time of PD

b : every other cycle (every 8 weeks)

${ }^{\mathrm{C}}$ : during Follow-up CTs or MRIs will be performed every 8 weeks ( \pm 7 days) until confirmed disease progression or death in the context of standard care.

$\mathrm{d}$ : at FU1 and time of PD

$\mathrm{e}_{\text {: at baseline (C1D1) }}$

f: only Arm B \&C

g: after 2 cycles for durvalumab maintenance

Figures 


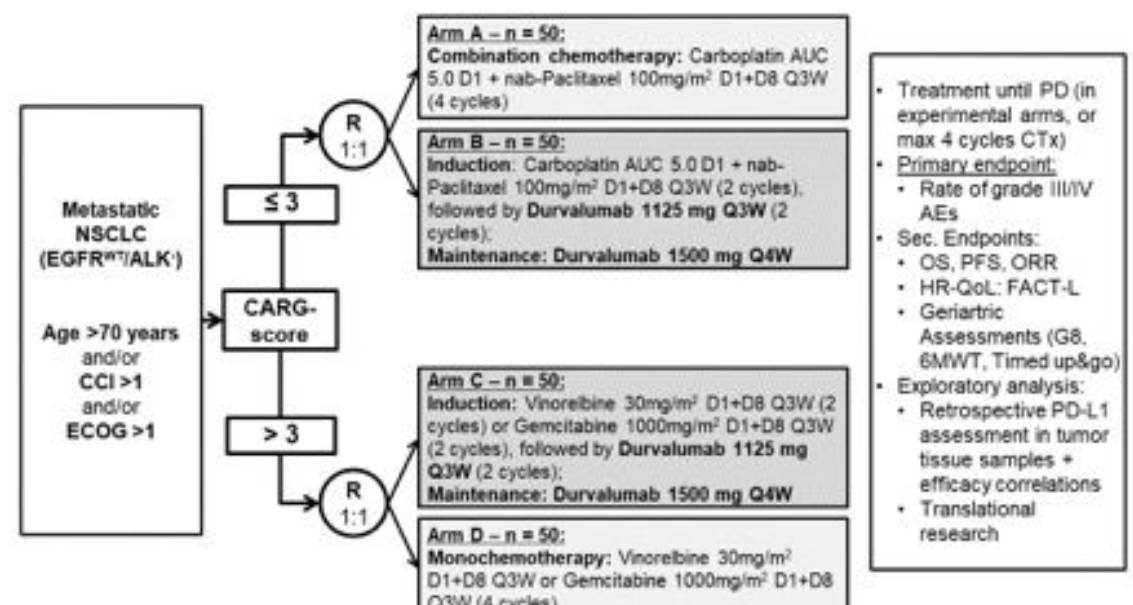

Figure 1

DURATION - Patient allocation and treatment strategy

\section{Supplementary Files}

This is a list of supplementary files associated with this preprint. Click to download.

- SPIRITChecklistDURATIONfinal.doc 